Case Report

\title{
Management of a Pregnancy with a Solid Pseudopapillary Neoplasm of the Pancreas
}

\author{
Atakan Tanacan $\mathbb{D}^{1},{ }^{1}$ Gokcen Orgul ${ }^{D},{ }^{1}$ Ahmet Bülent Dogrul, ${ }^{2}$ Fatih Aktoz, ${ }^{1}$ \\ Osman Abbasoglu, ${ }^{2}$ and M. Sinan Beksac ${ }^{1}$ \\ ${ }^{1}$ Division of Perinatology, Hacettepe University Medical Faculty, Ankara, Turkey \\ ${ }^{2}$ Department of General Surgery, Hacettepe University Medical Faculty, Ankara, Turkey
}

Correspondence should be addressed to Atakan Tanacan; atakantanacan@yahoo.com

Received 8 February 2018; Accepted 13 March 2018; Published 16 April 2018

Academic Editor: Giovanni Monni

Copyright (c) 2018 Atakan Tanacan et al. This is an open access article distributed under the Creative Commons Attribution License, which permits unrestricted use, distribution, and reproduction in any medium, provided the original work is properly cited.

\begin{abstract}
A 26-year-old primigravid patient, at 35 weeks and 2 days of gestation, was referred to Hacettepe University Hospital for pancreatic mass, giant cervical myoma, maternal systemic lupus erythematosus, thrombocytopenia, and onset of preterm labor. At 36 weeks and 1 day of gestation (6 days after admission to the hospital), regular uterine contractions started and cervical dilatation with effacement was observed. Because of breech presentation and giant cervical myoma, a cesarean section was performed on the primigravid patient under general anesthesia. Four months after the birth, subtotal pancreatectomy, partial gastrectomy, duodenectomy, cholecystectomy, and omentectomy (Whipple procedure) were performed. The pathologic diagnosis was of a solid pseudopapillary neoplasm of the pancreas; the patient was discharged from hospital after ten days.
\end{abstract}

\section{Introduction}

More than fifty percent of pancreatic cysts are composed of pancreatic cystic neoplasms (PCNs) $[1,2]$. PCNs are categorized by the World Health Organization (WHO) histologic classification system into four subtypes, as follows: (1) serous cystic tumors (SCTs), (2) mucinous cystic neoplasms (MCNs), (3) intraductal papillary mucinous neoplasms (IPMNs), and (4) solid pseudopapillary neoplasms (SPNs). SPNs account for 3 percent of PCNs [3]. SPNs are symptomatic in nearly half of cases, and the most common symptoms are abdominal pain, nausea, vomiting, and weight loss [4].

SPNs reveal themselves in cross-sectional imaging modalities as mixed solid and cystic pancreatic lesions and well-demarcated, echo-poor, solid, or solid-cystic lesions in ultrasound examinations [5]. Tumor markers, like carcinoembryonic antigen (CEA), can be used for differential diagnosis, especially for MCNs. Pathological tissue specimens must be taken for definitive diagnosis [6]. SPNs are rare and arise mostly in young women [7]. SPNs have a low malignancy potential, but surgical resection is recommended because they can be locally invasive and can spontaneously rupture.
In addition, distant organ and lymph node metastases can be seen in $5-10 \%$ and $2 \%$ of cases, respectively [8]. Even in the presence of malignancy complete excision and surgical debulking can provide a cure for the disease and prolong survival $[7,9,10]$.

SPNs can also complicate pregnancy [8, 11-13]. However, there is limited information related to adverse pregnancy outcomes and SPNs. In this case report, we presented the management of a pregnancy that was complicated by an SPN.

\section{Case Report}

A 26-years-old primigravid patient at 35 weeks and 2 days of gestation was referred to Hacettepe University Hospital for a pancreatic mass, giant cervical myoma, maternal systemic lupus erythematosus (SLE), thrombocytopenia, and onset of preterm labor. Ultrasound examination revealed a fetus appropriate for gestational age and a $20 \mathrm{~cm} \times 19 \mathrm{~cm} \times 22 \mathrm{~cm}$ cervical myoma. The patient was diagnosed with SLE in 2014, and she was taking $200 \mathrm{mg} /$ day hydroxychloroquine twice daily and $7.5 \mathrm{mg} /$ day Deltacortril. The patient was in SLE remission at the time of admission to the hospital. 


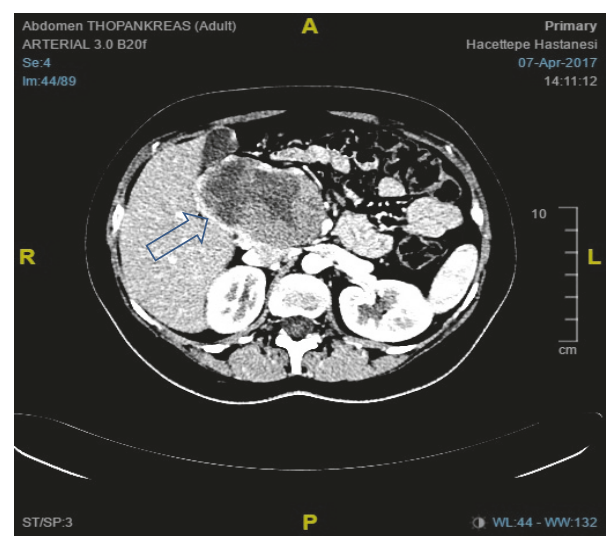

FIGURE 1: Contrast-enhanced CT of the abdomen showing the mass at the pancreatic head (arrow).

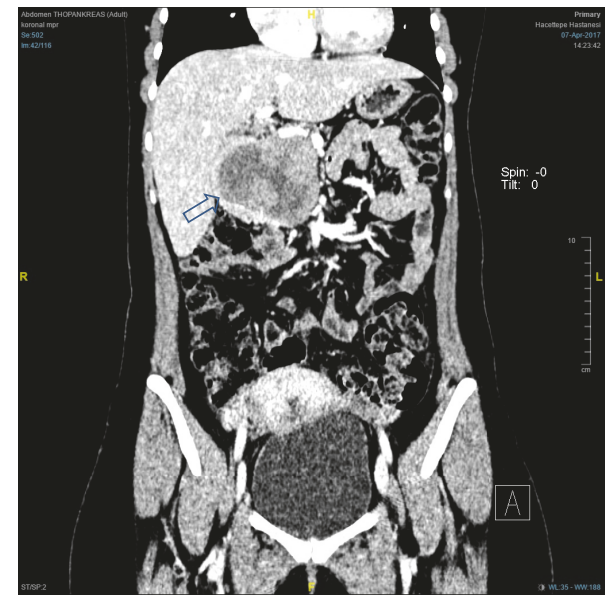

FIGURE 2: Coronal section of the pancreatic tumor (arrow).

At sixteen weeks of gestation, the patient presented at the hospital with abdominal pain, constipation, and abdominal distention. Abdominal ultrasound examination revealed a mass on the pancreas. Magnetic resonance imaging (MRI) of the pancreas was performed, and the result was reported as " $9 \mathrm{~cm} \times 7 \mathrm{~cm} \times 6 \mathrm{~cm}$ neoplasm at the head of the pancreas, which might be compatible with pseudopapillary neoplasm or less probably with cyst adenoma" (Figures 1 and 2). Management of the patient was discussed in a multidisciplinary medical council which consisted of radiologists, oncologists, perinatologists, general surgeons, and neonatologists. The council decided to postpone surgery in order to stabilize the patient's SLE and to prevent iatrogenic preterm delivery. At that time, there were low amplitude contractions of the uterus, with a frequency of four contraptions every ten minutes. Routine laboratory tests found only a low platelet count of $81 \times 10^{3} / \mathrm{mm}^{3}$. The patient consulted the rheumatology, general surgery, and cardiology departments before delivery. Four units of erythrocyte suspension (ES), 4 units of fresh frozen plasma (FFP), and 8 units of random thrombocyte (RT) suspensions were prepared. The patient was treated with intravenous hydration and bed rest in the obstetrics ward.

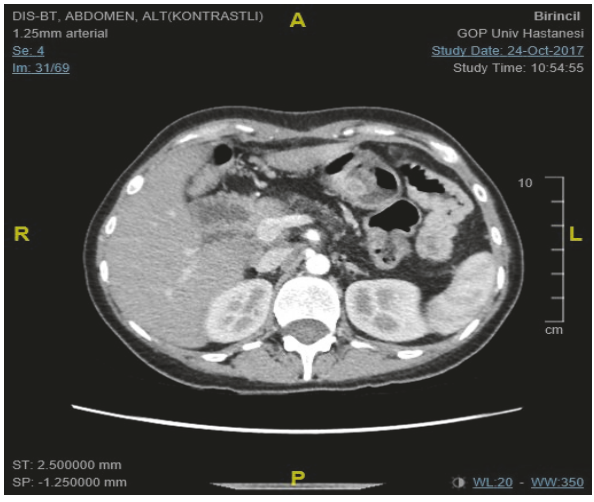

FIGURE 3: Contrast-enhanced computed tomography 7 months after Whipple procedure. No residual tumor can be seen.

At 36 weeks and 1 day of gestation ( 6 days after her admission to the hospital), regular uterine contractions started and cervical dilatation with effacement was observed. Due to breech presentation and giant cervical myoma, cesarean section (CS) was performed under general anesthesia. Laparotomy was performed with a Pfannenstiel incision, and CS was performed using a modified anterior transverse incision above the $20 \mathrm{~cm}$ myoma and a $3260 \mathrm{~g}$ male fetus was delivered with a five-minute APGAR score of 9 . A $20 \mathrm{~cm}$ cervical myoma was removed during the CS. Three units of ES, 4 units of FFP, and 8 units of RT were administered intraoperatively. After CS, the patient was treated at the hospital for a week and was discharged after general surgeons completed the plan for her subsequent surgery. The newborn was also discharged from the hospital without any complications.

Four months after the delivery, subpartial pancreatectomy, partial gastrectomy, duodenectomy, cholecystectomy, and omentectomy (Whipple procedure) were performed at the Department of General Surgery, Hacettepe University. The pathologic diagnosis was solid pseudopapillary neoplasm of the pancreas. The patient was discharged from the hospital after ten days of care. During a follow-up period of 7 months, the patient was tumor-free and in good health, without any complaints (Figure 3).

\section{Discussion}

SPNs of the pancreas are rare (2\% of pancreatic tumors) and mostly affect young women in their second and third decades of life [13]. Diagnosis can be challenging, as patients present with nonspecific symptoms [13]. SPNs have low malignancy potential but can be locally invasive and spontaneously rupture. Distant organ and lymph node metastases can be seen in only $5-10 \%$ and $2 \%$ of cases, respectively [8]. Thus, surgical intervention is recommended for definitive diagnosis and prevention of complications $[7,9,10]$.

Management of SPNs is more difficult during pregnancy because a balance between maternal and fetal well-being and surgical intervention for the tumor must be considered. Clinical findings, radiologic screening results, general surgery consultation, and tumor complications, along with maternal 
and fetal well-being, should all be assessed to determine the optimal timing of surgery. In our case, surgical intervention was postponed due to lack of sufficient evidence for the diagnosis of a malignant tumor. There is also limited clinical experience described in the literature due to the rarity of pancreatic tumors in pregnancy.

Yee et al. reported on a 39-year-old patient who was incidentally diagnosed with a large solid and cystic mass in the region of the pancreas at the eighteenth week of gestation. Noncontrast MRI identified a neoplasm at the head of the pancreas which was consistent with SPN. The patient was closely monitored for tumor growth by serial ultrasound screenings and she was delivered at term by spontaneous vaginal birth without complications. She underwent a pylorus-preserving Whipple procedure 3 months after the delivery, and the pathologic evaluation was found to be consistent with SPN. The patient was discharged from the hospital without any complication and the physicians detected no evidence of disease during her follow-up [8].

Huang et al. reported on a 29-year-old patient who was admitted to the hospital with a pancreatic mass and upper abdominal pain radiating to the back. Hypovolemic shock and peritoneal irritation signs emerged during her hospitalization. Subtotal pancreatectomy was performed for tumor bleeding and SPN was confirmed by pathology. The patient was delivered vaginally at term and no complications developed during the perinatal period. Both the mother and the infant were reported as healthy in their clinical follow-up eight months after the delivery [11].

Levy et al. reported a patient with SPN presenting with hyperemesis gravidarum. The tumor was resected surgically at the sixteenth week of gestation without complication. However, the surgical intervention did not relieve the symptoms (abdominal pain, nausea, and vomiting). Nevertheless, she was delivered near term without any complication [12].

MacDonald et al. reported on a 24-year-old patient with asymptomatic SPN diagnosed incidentally at 14 weeks of gestation. Distal pancreatectomy, splenectomy, and cholecystectomy were performed at 18 weeks of gestation. She delivered at term without complications [13].

In our case, the patient had SLE and a giant cervical myoma that complicated her pregnancy, but appropriate management by a multidisciplinary team provided optimal outcomes for both the mother and the infant. Although we have limited experience with SPNs in pregnancy, conservative management seems to be better in cases without tumor complications.

\section{Conflicts of Interest}

The authors declare that there are no conflicts of interest regarding the publication of this paper.

\section{References}

[1] K. S. Spinelli, T. E. Fromwiller, R. A. Daniel et al., "Cystic pancreatic neoplasms: observe or operate," Annals of Surgery, vol. 239, no. 5, pp. 651-659, 2004.
[2] C. Fernández-Del Castillo, J. Targarona, S. P. Thayer et al., "Incidental pancreatic cysts: Clinicopathologic characteristics and comparison with symptomatic patients," JAMA Surgery, vol. 138, no. 4, pp. 427-434, 2003.

[3] N. P. Valsangkar, V. Morales-Oyarvide, S. P. Thayer et al., "851 resected cystic tumors of the pancreas: a 33-year experience at the Massachusetts General Hospital," Surgery, vol. 152, no. 3, pp. S4-S12, 2012.

[4] L. Romics Jr., A. Oláh, T. Belágyi, N. Hajdú, P. Gyurus, and V. Ruszinkó, "Solid pseudopapillary neoplasm of the pancreasproposed algorithms for diagnosis and surgical treatment," Langenbeck's Archives of Surgery, vol. 395, no. 6, pp. 747-755, 2010.

[5] M. H. Yu, J. Y. Lee, M. A. Kim et al., "MR imaging features of small solid pseudopapillary tumors: retrospective differentiation from other small solid pancreatic tumors," American Journal of Roentgenology, vol. 195, no. 6, pp. 1324-1332, 2010.

[6] S. S. Vege et al., "American gastroenterological association institute guideline on the diagnosis and management of asymptomatic neoplastic pancreatic cysts," Gastroenterology, vol. 148, no. 4, pp. 819-822, 2015.

[7] J. K. Law, A. Ahmed, V. K. Singh et al., "A systematic review of solid-pseudopapillary neoplasms: are these rare lesions?" Pancreas, vol. 43, no. 3, pp. 331-337, 2014.

[8] A. M. Yee et al., "Solid pseudopapillary neoplasm of the pancreas head in a pregnant woman: safe pancreaticoduodenectomy postpartum," Journal of Surgical Case Reports, vol. 8, 2015.

[9] X. Chen et al., "Diagnosis and treatment of solid-pseudopapillary tumors of the pancreas," Hepatobiliary Pancreat Dis Int, vol. 4, no. 3, pp. 456-459, 2005.

[10] D. T. Alexandrescu, K. O. Boyle, A. Feliz, A. Fueg, and P. H. Wiernik, "Metastatic solid-pseudopapillary tumour of the pancreas: Clinico-biolgical correlates and management," Clinical Oncology, vol. 17, no. 5, pp. 358-363, 2005.

[11] S.-C. Huang et al., "Spontaneous rupture of solidpseudopapillary neoplasm of the pancreas during pregnancy," Obstetrics \& Gynecology, vol. 121, pp. 486-488, 2013.

[12] C. Levy et al., "Solid pseudopapillary pancreatic tumor in pregnancy. A case report," The Journal of reproductive medicine, vol. 49, no. 1, pp. 61-64, 2004.

[13] F. MacDonald, V. Keough, W.-Y. Huang, and M. Molinari, "Surgical therapy of a large pancreatic solid-pseudopapillary neoplasm during pregnancy," BMJ Case Reports, 2014. 


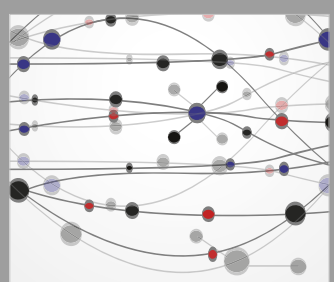

The Scientific World Journal
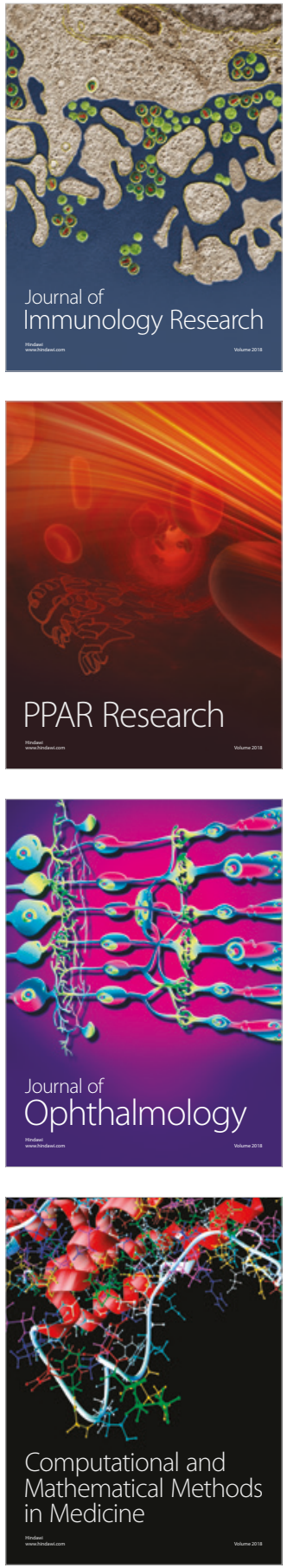

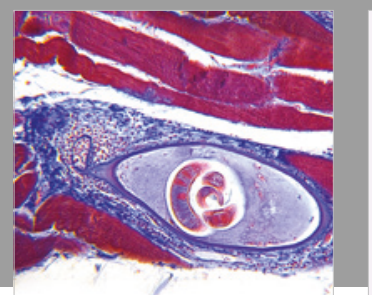

Gastroenterology Research and Practice

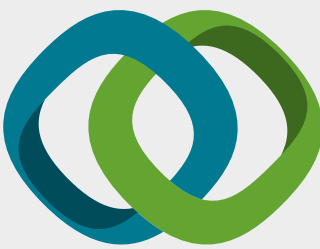

\section{Hindawi}

Submit your manuscripts at

www.hindawi.com
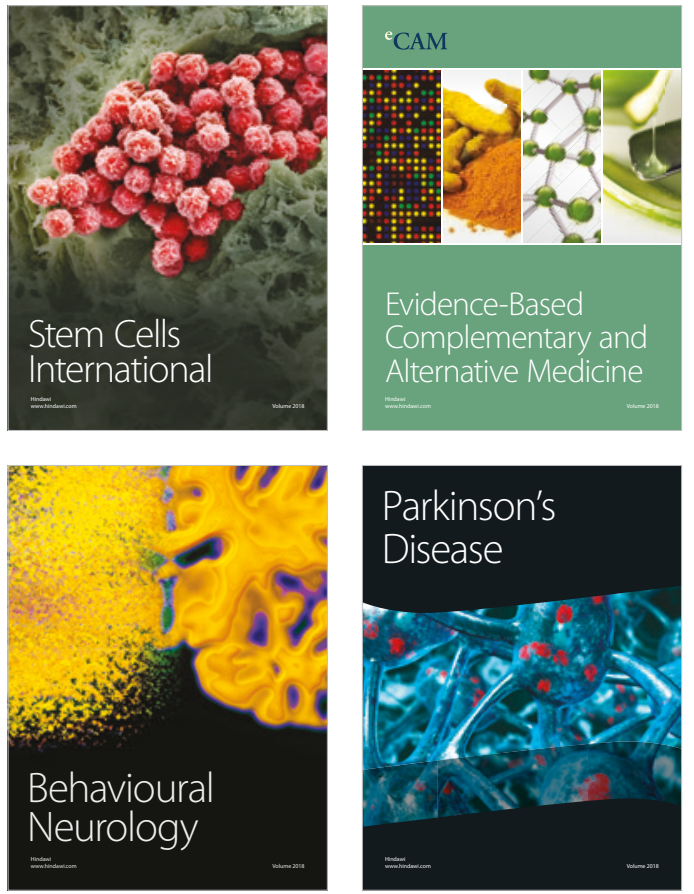

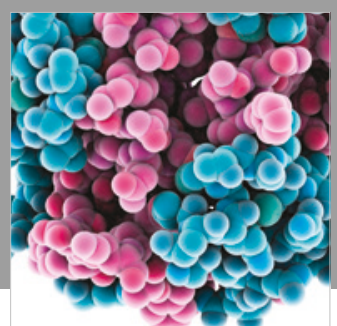

ournal of

Diabetes Research

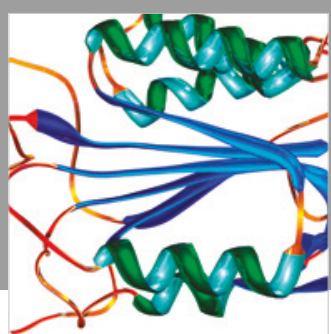

Disease Markers
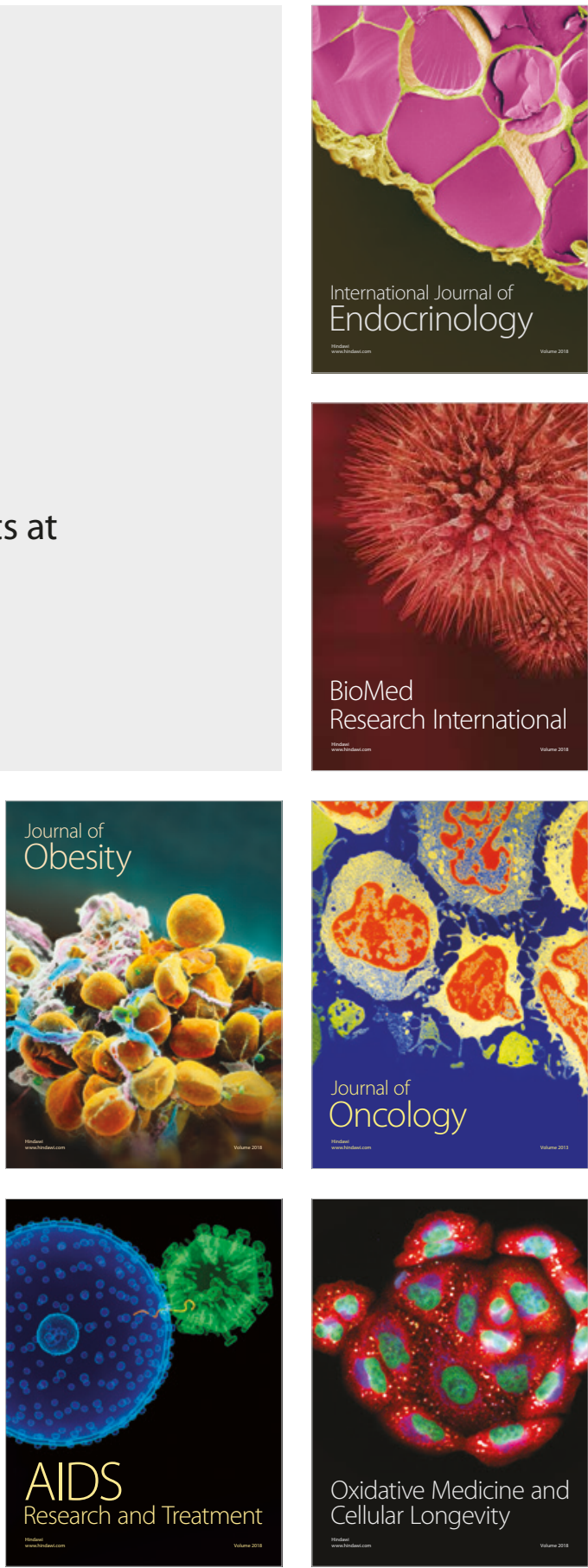